\title{
Trends in Healthcare Costs for Inflammatory Bowel Disease in South Korea
}

\author{
Yoon Suk Jung \\ Division of Gastroenterology, Department of Internal Medicine, Kangbuk Samsung Hospital, Sungkyunkwan University School of Medicine, \\ Seoul, Korea \\ See "Changes in Direct Healthcare Costs before and after the Diagnosis of Inflammatory Bowel Disease: A Nationwide \\ Population-Based Study" by Jooyoung Lee, et al. on page 89, Vol. 14, No. 1, 2020
}

Because inflammatory bowel disease (IBD) is a chronic and incurable disease with early onset and low mortality, the economic burden of IBD is becoming an important public health issue. ${ }^{1}$ The incidence and prevalence of IBD has been highest in North America and Europe; IBD is much less common in Asia. ${ }^{2}$ However, the incidence and prevalence of IBD in Asia is increasing rapidly. ${ }^{2-4}$ In particular, the recent incidence of IBD in South Korea has been the highest in East Asia. ${ }^{3}$ Accordingly, the economic burden of IBD in South Korea is increasing and it is becoming an important concern.

In this issue of Gut and Liver, Lee et al. ${ }^{5}$ conducted a population-based study to evaluate the direct healthcare costs of IBD in South Korea, by using the National Health Insurance (NHI) database. This study included 34,167 patients with IBD (23,153 patients with Crohn's disease [CD] and 11,014 patients with ulcerative colitis [UC]). They reported that patients with IBD incurred the highest direct healthcare costs during the first year after diagnosis $(\$ 2,396)$, and patients with IBD had higher costs than individuals without IBD even before the diagnosis of IBD. They also showed that the costs for biologics for the treatment of IBD increased steeply over time, rising from $\$ 721$ in the first year after diagnosis to $\$ 1,250$ in the 3rd year after diagnosis. These results suggest that biologics could be the main driver of costs during the early period after IBD diagnosis. Considering that most IBD patients continue to use biologics, biologics will be the main driver of IBD-related healthcare costs, not only during the early period after IBD diagnosis but also throughout the duration of the disease.

Although Lee et al. ${ }^{5}$ assessed the changes in costs before and after IBD diagnosis, they only analyzed medical costs for three years after diagnosis. Therefore, there was a limitation in identifying long-term trends in medical costs after IBD diagnosis. In addition, they analyzed only biologics and did not consider 5-aminosalicylic acid (5-ASA) or immunomodulators (azathioprine, 6-mercaptopurine), which are essential drugs for the treatment of IBD and may be important drivers of medical costs.

Very recently, another Korean study on a similar topic examined the medical costs of Korean patients with IBD for a longer period of time. ${ }^{6}$ This study was also conducted using NHI claims data. ${ }^{6}$ This population-based study included 59,447 patients with IBD (CD: 17,677; UC: 41,770) between 2006 and 2015, and demonstrated that explosive costs associated with IBD were due to the increased usage of anti-tumor necrosis factor (antiTNF) agents over the 10-year study period. ${ }^{6}$ More specifically, anti-TNF drugs accounted for 84.5\% (UC) and 93.1\% (CD) of all medication cost, and for $48.8 \%$ (UC) and $68.8 \%$ (CD) of the total cost in 2015. Furthermore, anti-TNF use was an independent predictor of increased total IBD cost and it was also the strongest predictor of high cost.

Both studies show that the increased use of biological agents has been the key driver of IBD-related healthcare costs. ${ }^{5,6}$ Biologic therapy with anti-TNF agents such as infliximab and adalimumab has revolutionized the treatment of IBD for their remarkable efficacy. ${ }^{7}$ For many years, these anti-TNF agents have become the mainstay of treatment for moderately or severely active IBD patients refractory or intolerant to conventional therapy. ${ }^{7,8}$ However, new biological and chemical drugs with different mechanisms of action from TNF blockade have been developed as potential therapeutic options for IBD, and have recently been emerging on the market. ${ }^{9,10}$ In addition to

\footnotetext{
Correspondence to: Yoon Suk Jung

Division of Gastroenterology, Department of Internal Medicine, Kangbuk Samsung Hospital, Sungkyunkwan University School of Medicine, 29 Saemunan-ro, Jongno-gu, Seoul 03181, Korea

Tel: +82-2-2001-8577, Fax: +82-2-2001-2049, E-mail: ys810.jung@samsung.com pISSN 1976-2283 eISSN 2005-1212 https://doi.org/10.5009/gnl19374

(a) This is an Open Access article distributed under the terms of the Creative Commons Attribution Non-Commercial License (http://creativecommons.org/licenses/by-nc/4.0) which permits unrestricted non-commercial use, distribution, and reproduction in any medium, provided the original work is properly cited.
} 
anti-TNF agents including infliximab, adalimumab, and golimumab, new drugs have recently been approved for the treatment of IBD in South Korea. More specifically, vedolizumab (antibody to $\alpha 4 \beta 7$-integrin), ustekinumab (antibody to interleukin-12/ interleukin-23 [p40]), and tofacitinib (oral small molecule Janus kinase inhibitor) have been approved since 2017 for the treatment of IBD in South Korea. The increase in these approved new drugs may further increase the healthcare cost burden for patients with IBD in the future. In this situation, long-term cost-saving strategies are imperative for patients with newly diagnosed IBD to balance the distribution of limited healthcare resources.

In order to make these strategies, further studies need to provide information such as the appropriate selection of patients who should receive biological therapy, the optimal timing of biologics initiation, and the appropriate choice of the first-line biologic for each patient. In addition, "exit strategies" such as de-escalating or stopping biologics in patients with IBD in remission should be considered. However, "exit strategies" should be carefully considered only for properly selected patients. In the near future, cost-cutting strategies optimized for Korean IBD patients should be proposed, particularly with a focus on reducing the use of biologics.

\section{CONFLICTS OF INTEREST}

No potential conflict of interest relevant to this article was reported.

\section{ORCID}

Yoon Suk Jung https://orcid.org/0000-0002-1963-7170

\section{REFERENCES}

1. Lee SH, Kwon JE, Cho ML. Immunological pathogenesis of inflammatory bowel disease. Intest Res 2018;16:26-42.

2. Ng WK, Wong SH, Ng SC. Changing epidemiological trends of inflammatory bowel disease in Asia. Intest Res 2016;14:111-119.

3. Jung YS, Han M, Kim WH, Park S, Cheon JH. Incidence and clinical outcomes of inflammatory bowel disease in South Korea, 2011-2014: a nationwide population-based study. Dig Dis Sci 2017;62:2102-2112.

4. Yen HH, Weng MT, Tung CC, et al. Epidemiological trend in inflammatory bowel disease in Taiwan from 2001 to 2015: a nationwide populationbased study. Intest Res 2019;17:54-62.

5. Lee J, Im JP, Han K, et al. Changes in direct healthcare costs before and after the diagnosis of inflammatory bowel disease: a nationwide population-based study. Gut Liver 2020;14:89-99.

6. Kim JW, Lee CK, Lee JK, et al. Long-term evolution of direct healthcare costs for inflammatory bowel diseases: a populationbased study (2006-2015). Scand J Gastroenterol 2019;54:419-426.

7. Im JP, Ye BD, Kim YS, Kim JS. Changing treatment paradigms for the management of inflammatory bowel disease. Korean J Intern Med 2018;33:28-35.

8. Ooi CJ, Hilmi I, Banerjee R, et al. Best practices on immunomodulators and biologic agents for ulcerative colitis and Crohn's disease in Asia. Intest Res 2019;17:285-310.

9. Na SY, Moon W. Perspectives on current and novel treatments for inflammatory bowel disease. Gut Liver 2019;13:604-616.

10. Katsanos KH, Papadakis KA. Inflammatory bowel disease: updates on molecular targets for biologics. Gut Liver 2017;11:455-463. 